\title{
Modeling of Shock Wave Propagation through Energetic Solid State Composites using a Taylor-Galerkin Scheme
}

\author{
Adam V. Duran,* \\ University of Michigan, Ann Arbor, Michigan, 48109, USA \\ Veera Sundararaghavan ${ }^{\dagger}$ \\ University of Michigan, Ann Arbor, Michigan, 48109, USA
}

\begin{abstract}
A structural dynamics code for modeling extreme events such as shock and detonation waves in Polymer-Bonded Explosives (PBXs)is presented. PBXs are multi-component solid-state composite consisting of energetic crystals and metal particles embedded in a polymeric binder and are used for conventional munitions. A stable efficient solution strategy based on a Taylor-Galerkin finite element (FE) discretization was developed to solve PBXs behavior under extreme shock loading. Equations of state for the solid unreacted material and gaseous reaction products are implemented using a pressure mixture rule governed by pressure based reaction rates. The FE model is verified using analytical solutions for Sod shock and ZND strong detonation models. Numerical results for PBXs under shock loading are presented using a continuum model and a first-order multiscale model.
\end{abstract}

\section{Introduction}

Energetic composite materials are used as propellants, explosives and fuel cell components. The rapid energy release (detonation) of these materials involve tightly coupled nonlinear interactions between chemistry and mechanics. During detonation, a shock wave is sustained by the rapid chemical energy heat release from the energetic material. These waves have extreme features; they travel at typical speeds of several thousand meters per second and the peak pressures can reach about $100 \mathrm{GPa} .{ }^{1}$ Laboratory experiments are seldom equipped to handle these events, where safety and performance are paramount. Therefore, there is significant interest in engineering the microstructures of these energetic composites for targeted shock sensitivity and energy output. Literature in this area indicate the importance of composite features, for example, smaller energetic particles have lesser run time to detonation ${ }^{2}$ and the time to detonation increases with the strength and content of the matrix (binder) material. ${ }^{3}$ The first step in understanding the effects of the microstructure is to develop a reliable computational model for PBXs at the meso-scale.

The evolution of the solid state composite during detonation can be computed by solving the reactive Euler equations, a non-linear set of hyperbolic equations. In such equations, discontinuities (shocks) arise from continuous initial conditions, that can be found by solving a weak form of the PDE. Classical formulations of solid dynamics based on displacement approach are unsuitable for this problem due to locking issues at extreme mesh distortions and numerical errors in the velocity of propagation of waves. This problem has been largely addressed in the fluid dynamics community using Riemann solvers, albeit mostly in the context of Eulerian finite volume methods. ${ }^{4-9}$ In the context of standard finite element methods, various stabilized methods such as Petrov Galerkin (PG) methods, Galerkin/least-squares (GLS) methods and the Taylor-Galerkin (TG) methods, have been developed to address the issue. In the PG and GLS methods, a stabilization term with a coefficient is added to the weak form to act as an artificial diffusion, however, the choice of the coefficient is semi-empirical and may lead to overdamping. ${ }^{10,11}$ The basic TG algorithm

*PhD Candidate, Department of Aerospace Engineering, avduran@umich.edu, Student Member.

†Associate Professor, Department of Aerospace Engineering, veeras@umich.edu, AIAA Senior Member. 
was proposed by Donea, ${ }^{12}$ Baker et $\mathrm{al}^{13}$ and Löhner et $\mathrm{al}^{14}$ for first-order systems of hyperbolic equations in which Taylor expansion in time precedes the Galerkin space discretization. TG finite element schemes are especially attractive since the scheme has no free parameter, in fact, the diffusion arises from an improved Taylor approximation to the time derivative of the fields. While TG algorithms have been successfully applied in areas such as pollutant transport, shallow water problems and fluid dynamics, ${ }^{15-17}$ there do not exist a prior study of the technique for reactive euler equations.

A variety of different reaction-rate equations are used in reactive-burn models. Arrhenuis reactive-burn models use reaction schemes that are tuned to experimental data and chemical data such as heats of formation. Reaction kinetics of PBXs involve three general steps (i) endothermic dissociation (ii) formation of reactive intermediates and (iii) exothermic recombination. Intermediate species only exist for a few nanoseconds and are extremely difficult to measure experimentally. Hence, reaction kinetics are often approximated in a one step form ${ }^{4}$ of solid reactant state to gaseous product state, although other schemes have been proposed with three-step ${ }^{18}$ four-step ${ }^{19}$ or multi-step reactions. ${ }^{20}$ These schemes typically require thermal and pressure equilibrium. The Ignition and Growth model ${ }^{21}$ is a pressure-dependent model formulated to represent the ignition of hotspots and the growth of reaction from hotspots into the surrounding explosive and are based on empirical parameters. This model requires pressure equilibrium for the solid unreacted explosive and the reacted products. A simplified version of the Ignition and Growth model is the JWL++ model. ${ }^{22}$ The JWL++ model uses a mixture rule for the total pressure and has found that it is equivalent to enforcing pressure equilibrium.

The equation of state needs to be represented for each of the reaction states. A variety of equations of state (EOS) have been used to represent explosives, the popular ones being the Jones-Wilkins-Lee (JWL) form, ${ }^{21}$ the Murnaghan form ${ }^{22}$ and Gruneisen form. ${ }^{5}$ The Gruneisen from with a linear shock velocity versus particle velocity Hugoniot has been employed in several studies. ${ }^{5,23,24}$ For the gaseous reaction products, the by far the most popular equation of state is the JWL form that was developed by measuring the expansion velocity of metal casings surrounding HMX. ${ }^{25}$ This work uses the Murnaghan equation of state for the unreacted solid and the C-form JWL equation of state for the reacted detonation products. In this paper, computational modeling of reactive Euler equations is presented using the TG approach as applied to detonation of PBXs in 1D and 2D.

\section{Governing Equations}

\section{A. Euler Equations}

The 1-D reactive Euler equations are given by the following vector equations. Here, $\rho$ is the density, $\rho u$ is the momentum and $\rho E$ is the total energy per unit volume. The subscripts $x$ and $t$ denote partial derivatives. Diffusion, viscosity and radiation effects are neglected.

$$
U_{t}+F_{x}=S
$$

with

$$
U=\left\{\begin{array}{c}
\rho \\
\rho u \\
\rho E \\
\rho \lambda
\end{array}\right\} \quad F=\left\{\begin{array}{c}
\rho u \\
\rho u^{2}+p \\
(\rho E+p) u \\
\rho u \lambda
\end{array}\right\} \quad S=\left\{\begin{array}{c}
0 \\
0 \\
0 \\
\rho R(p, \lambda)
\end{array}\right\}
$$

The Euler equations are written in the quasi-linear form with jacobian matrices $A=\partial F / \partial U$. The flux vectors are rewritten as

$$
F=A U .
$$

The transport equation for the burn fraction $\lambda$ is governed by the source term modeling the reaction rate $R(p, \lambda)$. For a solid unreacted material $\lambda=0$ and for the completely reacted products $\lambda=1$. The reaction rate is given by the Ignition and Growth form in the following equation. Here, $I$ is the ignition parameter and $G$ is the growth parameter. The relative volume $\eta=\rho / \rho_{0}$ where $\rho_{0}$ is the reference density. The empirical parameters for this model are taken from Ref. ${ }^{21}$

$$
R(p, \lambda)=I(1-\lambda)^{x} \eta^{r}+G(1-\lambda)^{x} \lambda^{y} p^{z}
$$




\section{Numerical Scheme}

\section{A. One-step Taylor Galerkin Scheme}

The 1-D reactive Euler equations given by Eq.1 are solved using a one-step Taylor Galerkin scheme. This widely used time-stepping algorithm is second-order accurate, explicit and analogous to the Lax-Wendroff method. Taking a Taylor series expansion of $U$

$$
U^{n+1}=U^{n}+\Delta t U_{t}^{n}+\frac{1}{2} \Delta t^{2} U_{t t}^{n}+\mathcal{O}\left(\Delta t^{3}\right)
$$

where $\Delta t$ is the time step, $t^{n+1}$ is the current time and $t^{n}$ is the previous time. The first term of the RHS of Eq.5 is the state vector $U$ from Eq.2. The second term is found from rearranging Eq.1; i.e.

$$
U_{t}=-F_{x}+S
$$

The third term from the Taylor expansion is found by differentiating Eq.6 with respect to time. Now Eq.5 is written as

$$
U^{n+1}=U^{n}+\Delta t\left(S-F_{x}\right)^{n}+\frac{1}{2} \Delta t^{2}\left[S_{t}-\left(A S-A^{2} U_{x}\right)_{x}\right]^{n}
$$

The rate of the source term $S_{t}$ is found by finite differences on $S$ from Eq. 2 ; i.e. $S_{t}=\left(S^{n}-S^{n-1}\right) / \Delta t$. The associated variational form is given as follows after integration by parts of the spatial terms

$$
\begin{array}{rl}
\int_{0}^{L} W \frac{U^{n+1}-U^{n}}{\Delta t} d x=\int_{0}^{L} & W S^{n} d x+\int_{0}^{L} W_{x} F^{n} d x+\frac{1}{2} \Delta t \int_{0}^{L} W S_{t}^{n} d x \\
& +\frac{1}{2} \Delta t \int_{0}^{L} W_{x}\left(A S-A^{2} U_{x}\right)^{n} d x-\left[W \cdot\left(F^{n}+\frac{1}{2} \Delta t\left(A S-A^{2} U_{x}\right)^{n}\right)\right]_{x=0}^{x=L}
\end{array}
$$

Modifying the boundary term based on the relation $F_{t}^{n}=F_{u} U_{t}^{n}=A\left(S-F_{x}\right)^{n}=\left(A S-A^{2} U_{x}\right)^{n}$,

$$
\begin{aligned}
\int_{0}^{L} W \frac{U^{n+1}-U^{n}}{\Delta t} d x=\int_{0}^{L} W S^{n} d x+ & \int_{0}^{L} W_{x} F^{n} d x+\frac{1}{2} \Delta t \int_{0}^{L} W S_{t}^{n} d x \\
& +\frac{1}{2} \Delta t \int_{0}^{L} W_{x}\left(A S-A^{2} U_{x}\right)^{n} d x-\left[W \cdot\left(F^{n}+\frac{1}{2} \Delta t F_{t}^{n}\right)\right]_{x=0}^{x=L}
\end{aligned}
$$

In this work, flux representation depends on the compressibility of the fluid. In regions of compression where $\partial u / \partial x<0$ a group representation, shown in equation 10 is used, and the classical flux representation is used otherwise.

$$
F_{i}=\sum_{k=1}^{E L M} W_{k}(x, y) F_{i}\left(U_{k}\right), i=1,2 .
$$

\section{B. Numerical Treatment of Shocks}

A high-resolution scheme is used for the numerical treatment of shocks. ${ }^{26}$ This scheme is used for the purpose of adding minimal numerical dissipation and to give non-oscillatory solutions in the presence of steep solution gradients. Numerical dissipation is applied in the neighborhood of a discontinuity where the diffusion coefficient is calculated based on the local behavior of the solution. After Galerkin spatial discretization the following algebraic equation is obtained

$$
M\left(U^{n+1}-U^{n}\right)=\Delta t B^{n}
$$

where $M$ is the mass matrix and $B$ is the force vector. The consistent mass matrix $M$ acting on $U^{n+1}$ is replaced by the diagonal matrix $M^{L}$, obtained by row sum. Now Eq.11 becomes

$$
M^{L} U^{n+1}=M U^{n}+\Delta t B^{n}
$$


which can be rewritten as

$$
M^{L} U^{n+1}=M^{L} U^{n}+\left(M-M^{L}\right) U^{n}+\Delta t B^{n}
$$

where the term $\left(M-M^{L}\right) U^{n}$ represents added dissipation. This term is multiplied by the parameter $d$, where $0 \leq d \leq 1$; for maximum dissipation $d=1$. Now the system is written as

$$
M^{L} U^{n+1}=M^{L} U^{n}+d\left(M-M^{L}\right) U^{n}+\Delta t B^{n}
$$

For a value of $d>0$ the system adds dissipation to the entire system; i.e. global smoothing. By controlling $d$ locally, in the presence of shocks, we can perform local smoothing. The following two stage procedure is used where the first stage corresponds to the first-order Taylor-Galerkin scheme (Eq.11) and the second introduces dissipation

$$
\begin{gathered}
M\left(U^{*}-U^{n}\right)=\Delta t B^{n} \\
M^{L}\left(U^{n+1}-U^{*}\right)=d\left(M-M^{L}\right) U^{*}
\end{gathered}
$$

Locally, at a node " $i$ " the right hand side of Eq.16 is

$$
\sum_{j} d_{i j} M_{i j}\left(U_{j}^{*}-U_{i}^{*}\right)
$$

where nodes " $j$ " are connected to node " $i$ ". To detect the presence of shocks $d_{i j}$ is constructed by considering the second derivative of pressure.

$$
d_{i j}=\min \left[\chi \max \left(d_{i}, d_{j}\right), 1\right]
$$

where

$$
\begin{aligned}
d_{i} & =\left|\frac{p j-2 p i+p i_{-}}{p j+2 p i+p i_{-}}\right|, \\
d_{j} & =\left|\frac{p j_{+}-2 p j+p i}{p j_{+}+2 p j+p i}\right|
\end{aligned}
$$

and

$$
\begin{aligned}
& p i_{-}=p j-2(x j-x i) \cdot[\nabla p] i, \\
& p j_{+}=p i-2(x j-x i) \cdot[\nabla p] j .
\end{aligned}
$$

\section{Numerical Results for SOD Shock and ZND}

To test the stability and accuracy of the scheme described above, the classical fluid dynamics shock tube problem is solved. The test consists of two fluids at differing pressures separated by a membrane. Once the membrane is removed a rarefaction wave, contact discontinuity and shock wave is formed. The the solution for an ideal gas is obtained analytically using Riemann invariants and is compared with numerical results on Figures 1-3. The numerical results on Figures 1-3 show good agreement with the exact solution and the distinct characteristics of the test are captured. To verify that the numerical scheme described above is accurate for reactive flow, the ZND detonation model is tested. The model produces a von Neumann spike that propagates at a constant speed. Numerical results are compared with the exact solution on Figures 4 and 5 and are in good agreement.

\section{Continuum model of Polymer-Bonded Explosives}

It is necessary to represent the pressure of both the unreacted material and the reaction products of PBXs; however, is is also necessary to model the material when it is at neither extreme (ie $0<\lambda<1$ ). This

work follows the JWL ++ model. The Murnaghan equation is used for unreacted equation of state and is given by

$$
p=p_{s}=\frac{1}{n \kappa}\left(\frac{1}{\left(\rho_{0} / \rho\right)^{n}}-1\right)
$$




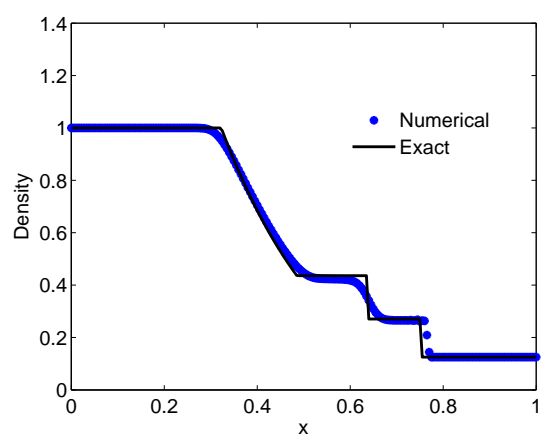

Figure 1. Shock tube density for ideal gas.

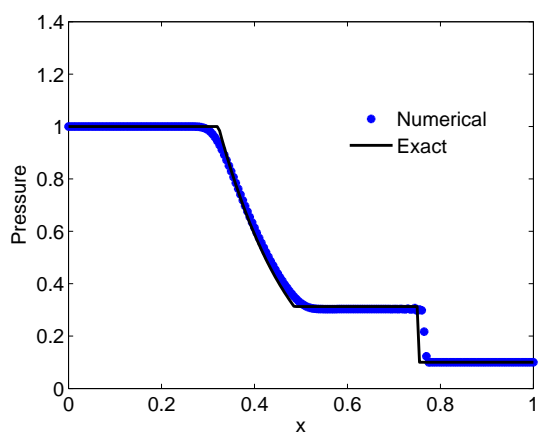

Figure 3. Shock tube pressure for ideal gas.

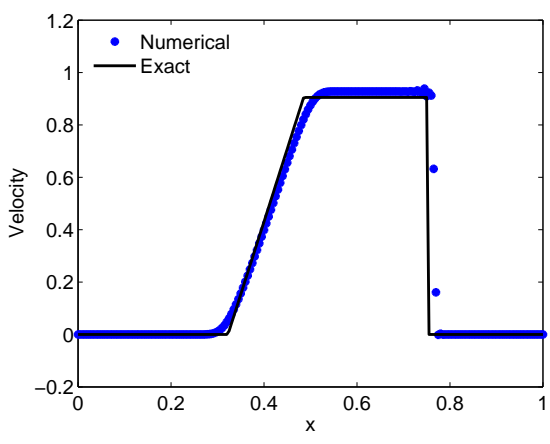

Figure 2. Shock tube velocity for ideal gas.

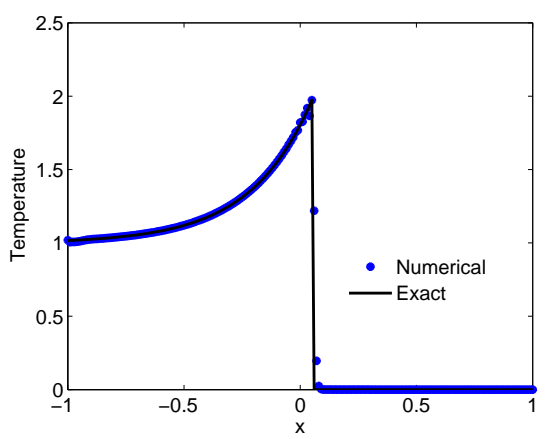

Figure 4. ZND mass fraction profile.

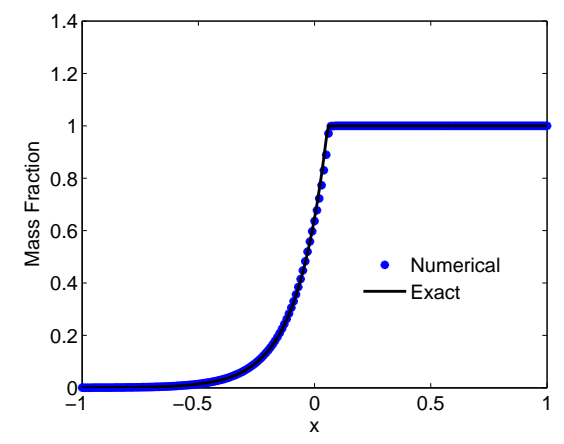

Figure 5. ZND mass fraction profile.

The parameters $n$ and $\kappa$ are derived from the linear shock relationship between shock velocity $U_{S}$ and particle velocity $u_{p}$. This Hugoniot data is available for a number of explosives. A JWL in the C-term form is used for the EOS of the fully reacted detonation products.

$$
p=p_{g}=A e^{-R_{1} \rho_{0} / \rho}+B e^{-R_{2} \rho_{0} / \rho}+\frac{C}{\left(\rho_{0} / \rho\right)^{1+\omega}}
$$

For the intermediate state, a pressure mixture rule is used to find the total pressure

$$
p=(1-\lambda) p_{s}+\lambda p_{g}
$$

The burn fraction $\lambda$ is governed by equation 4. All parameters for the equations of state are taken from experiments in Ref. ${ }^{27}$ for the explosive PBX 9404. To initiate the detonation wave in PBX 9404, the Hugoniot for the solid $\lambda=0$ and the reaction products $\lambda=1$ are computed on Figure 6 . Thermodynamics 
theory states that the material must be shocked to the Von Neumann pressure $p_{V N}$ in order to have a self-sustaining wave. The pressure then drops down the Rayleigh-line to the Chapman-Jouget pressure $p_{C J}$ the point tangent to the Hugoniot of the reaction products. The Von Neumman pressure was calculated by finding the intersection between the solid Hugoniot and the Rayleigh-line.

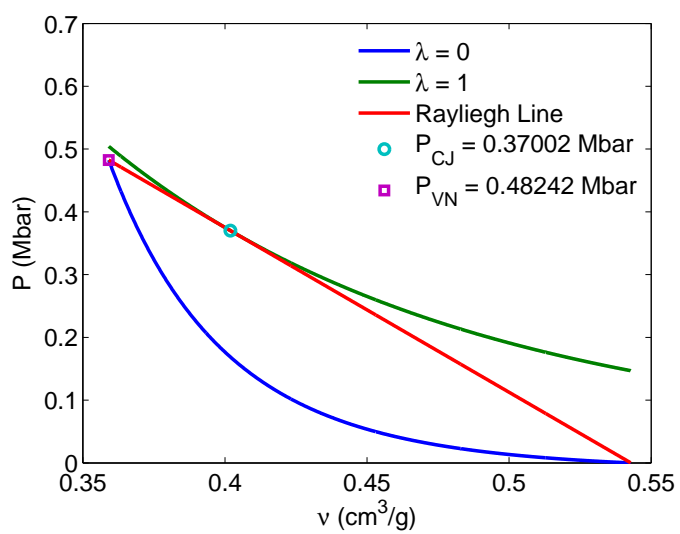

Figure 6. Hugoniot for PBX 9404.

Using the numerical scheme described in the sections above, detonation of PBX 9404 is performed. In the left side of the domain, the material is pressurized to the calculated Von Neumman pressure $p_{V N}=$ $0.482 \mathrm{Mbar}$ while the left side of the domain is left at ambient conditions where pressure is zero. A uniform mesh of 200 linear elements for a domain of $2.5 \mathrm{~cm}$ is used. The duration of the simulation is $t=2 \mu s$ with $\Delta t=5 e^{-4} \mu \mathrm{s}$. Results for density, velocity, burn fraction and pressure vs. distance at time-steps of $0.2 \mu \mathrm{s}$ are shown on Figures 7-10. All solutions show a self-sustaining wave propagate through the domain. Figure 7 shows density drop initially from the Von Neumman specific volume. This region begins to expand below the value of the reference density. Velocity, shown in Figure 8, is initially zero and increases to peak values around $3 \mathrm{~km} / \mathrm{s}$. The burn fraction $\lambda$ is initially set to a pure solid as shown in Figure 9 . As time increases the reaction occurs and the solid is burnt and completely reacted. There is an observable time disparity between the rate of reaction and shock speed. The pressure plotted in Figure 10 initially drops from the initial pressure $p_{V N}$. As the reaction initiates pressure recovers to $p_{V N}$ and a self-sustaining detonation progresses through the domain. However the value calculated at the peak of the pressure profile $p=0.475 \mathrm{Mbar}$ is under the calculated Von Neumman pressure. This can be attributed to the numerical scheme used to damp spurious oscillations near the shock front. The same experiment for the shocked PBX 9404 is performed in two-dimensions. A radial symmetric detonation waved is used with the initial discontinuity located at $r=0.5 \mathrm{~cm}$. The combustion front is a quarter circle that expands as the system evolves. A uniform mesh with a $200 X 200$ quadrilateral elements is used with $\Delta t=5 e^{-4} \mu s$ and no-slip boundary conditions. Numerical results for density and pressure are shown on Figures 11,12 and 13, 14 respectively. The detonation profile maintains a symmetric spherical shape through the experiment and is in good agreement with the 1D case.

\section{First-Order Multiscale Modeling of Polymer-Bonded Explosives}

The 1-D reactive Euler equations are updated to include multiscale state variables for HMX and binder. The transport equation for burn fractions $\lambda_{H, B}$, where $H$ denotes HMX and $B$ denotes binder, are governed by the source term modeling the reaction rate $R_{H, B}\left(\bar{p}, \lambda_{H, B}\right)$. For a solid unreacted material $\lambda_{H, B}=0$ and for the completely reacted products $\lambda_{H, B}=1$. Burn fractions for both HMX and binder are governed by the simple Growth model shown in Eq.25. Here, $G_{H, B}$ is the growth parameter and $b_{H, B}$ is the pressure exponent. The empirical parameters for this model are taken from Ref. ${ }^{21}$ 


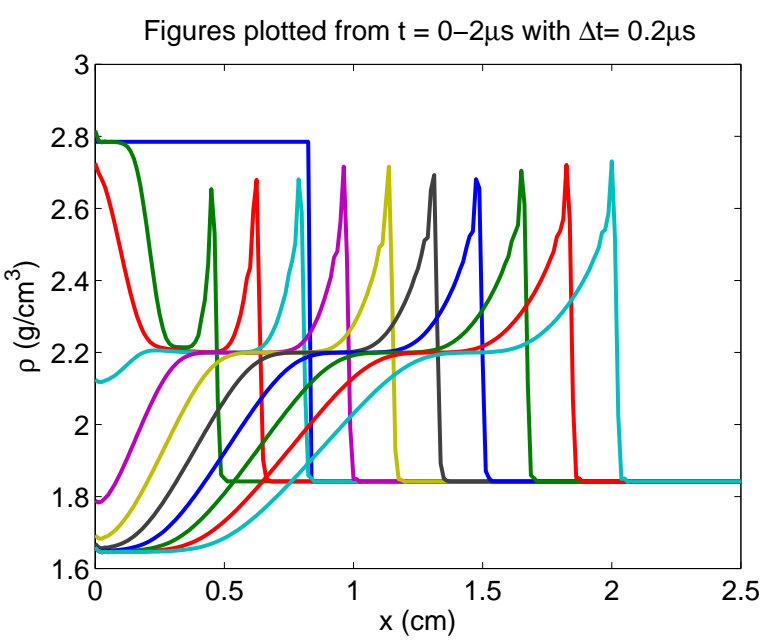

Figure 7. Density for PBX9404 at timesteps of $0.2 \mu \mathrm{s}$.

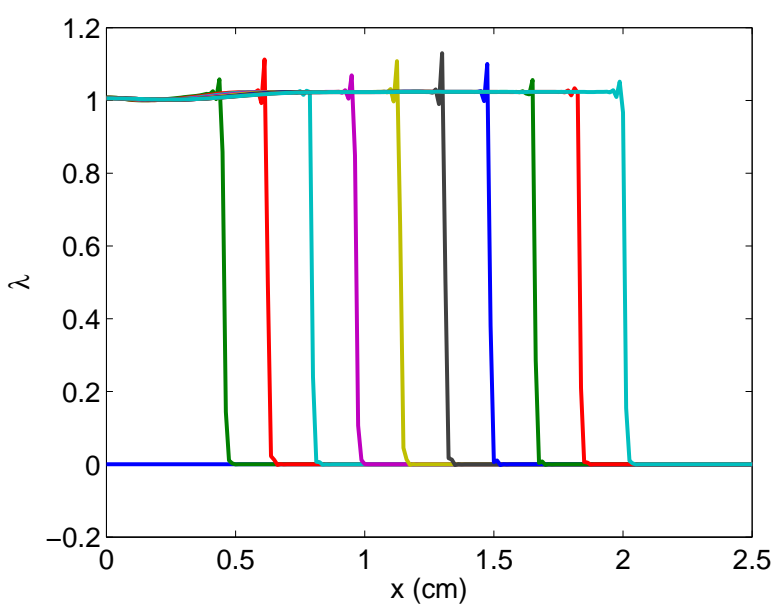

Figure 9. Burn fraction for PBX9404 at time-steps of $0.2 \mu \mathrm{s}$.

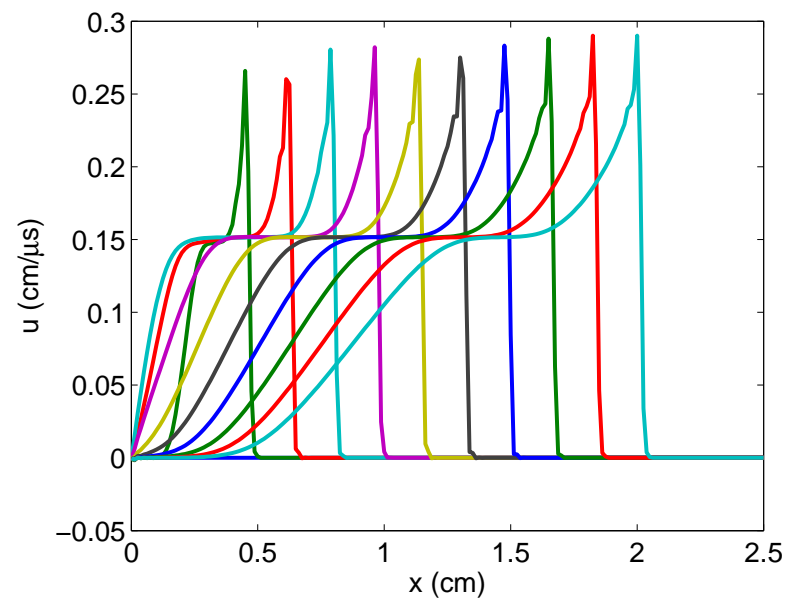

Figure 8. Velocity for PBX9404 at timesteps of $0.2 \mu \mathrm{s}$.

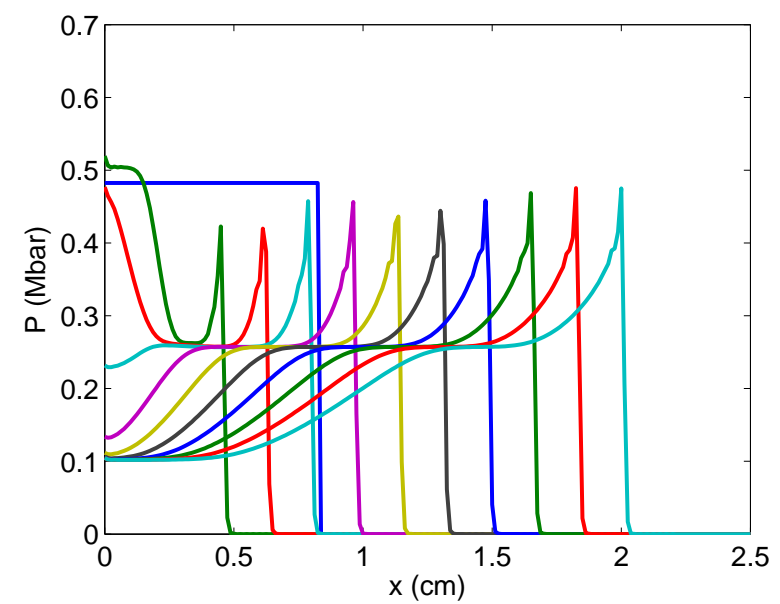

Figure 10. Pressure for PBX9404 at time-steps of $0.2 \mu \mathrm{s}$.

$$
\begin{gathered}
U=\left\{\begin{array}{c}
\rho \\
\rho u \\
\rho E \\
\rho \lambda_{H} \\
\rho \lambda_{B}
\end{array}\right\} \quad F=\left\{\begin{array}{c}
\rho u \\
\rho u^{2}+\bar{p} \\
(\rho E+\bar{p}) u \\
\rho u \lambda_{H} \\
\rho u \lambda_{B}
\end{array}\right\} \quad S=\left\{\begin{array}{c}
0 \\
0 \\
0 \\
\rho R_{H} \\
\rho R_{B}
\end{array}\right\} \\
R_{H, B}=G_{H, B}\left(1-\lambda_{H, B}\right) \bar{p}^{b_{H, B}}
\end{gathered}
$$

The total pressure of the composite $\bar{p}$, is determined by calculating pressures for both HMX and binder. Using the JWL ++ model, ${ }^{22}$ the Murnaghan equation is used for unreacted equation of state for both HMX and binder $\left(p_{S(H, B)}\right)$ and is given by Eq.21. A JWL in the C-term form is used for the EOS of the fully reacted detonation products for both HMX and binder $\left(p_{G(H, B)}\right)$ and is given by Eq.22. Parameters for HMX and for binder are taken from Ref. ${ }^{28}$ For the intermediate state a pressure mixture rule is used to find the total pressure.

$$
p_{H, B}=\left(1-\lambda_{H, B}\right) p_{S,(H, B)}+\lambda_{H, B} p_{G,(H, B)}
$$




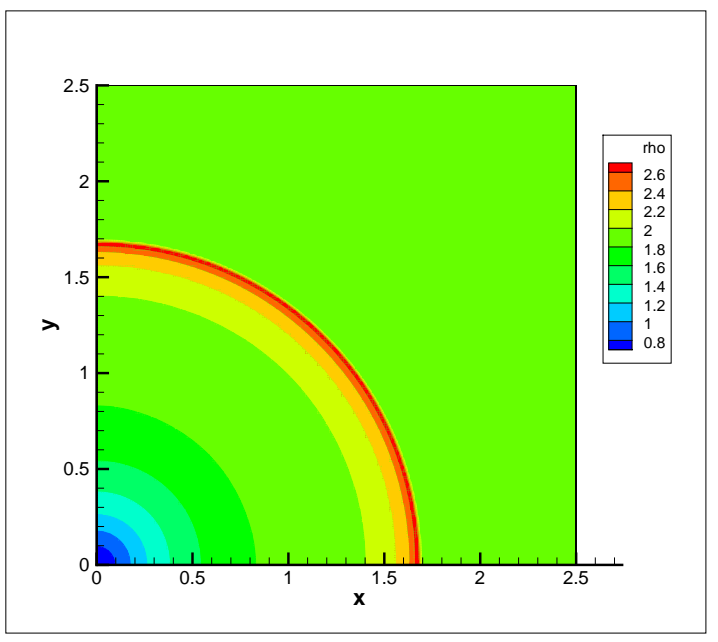

Figure 11. Density for PBX9404 at $t=$ $1 \mu s$.

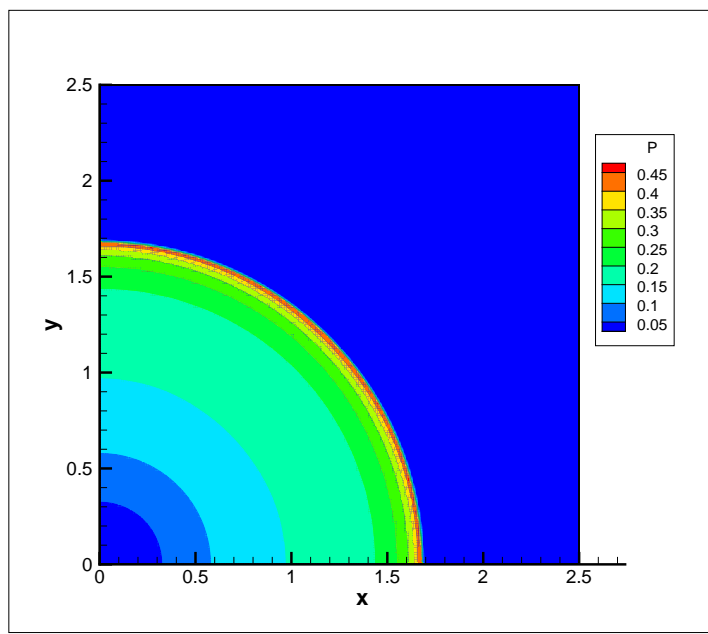

Figure 13. Pressure for PBX9404 at $t=$ $1 \mu s$.

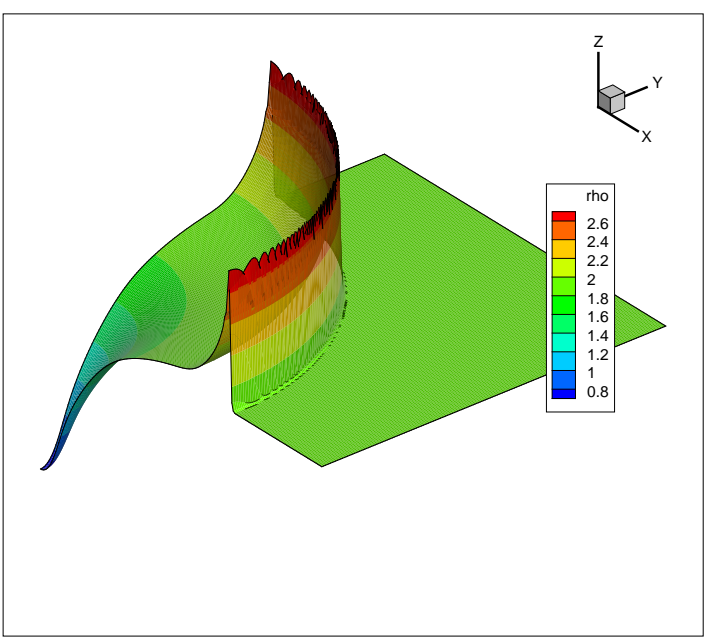

Figure 12. Density waterfall plot for $P B X 9404$ at $t=1 \mu s$.

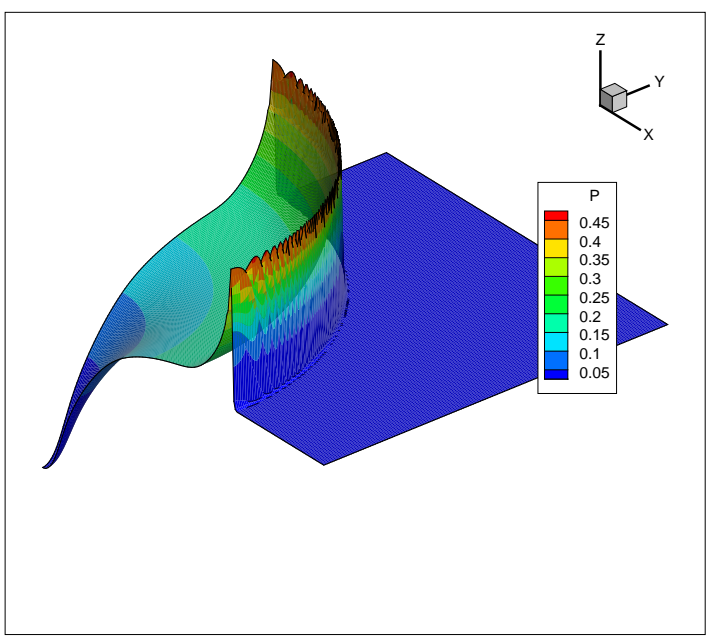

Figure 14. Pressure waterfall plot for $P B X 9404$ at $t=1 \mu s$.

The total mass is comprised of both $\operatorname{HMX} \rho_{H}$ and binder $\rho_{B}$. Here, $N_{H}$ represents the mass fraction of the energetic material HMX and $N_{B}$ represents the mass fraction of binder.

$$
\rho=\rho N_{H}+\rho N_{B}=\rho_{H}+\rho_{B}
$$

Now that the densities of HMX and binder are known, they are passed to the equations of state (Eq. 21,22) and the total pressures for both HMX $p_{H}$ and binder $p_{B}$ are calculated from Eq. 23. The total pressure of the composite is calculated by the following equation and is based on the mass fractions of each species. This methodology is described on Figure. 15.

$$
\bar{p}=p_{H} N_{H}+p_{B} N_{B}
$$

\section{Numerical Results}

For the following experiment, the continuum model uses a homogeneous medium described in Section $\mathrm{V}$ while the multiscale model has $5 \%$ binder explicitly modeled $\left(N_{H}=0.95\right.$ and $\left.N_{B}=0.05\right)$. Hugonoit data calculated for PBX9501 is shown on Figures 6 and 17. These figures compare the pressures calculated using the multiscale model described above(black plot) and a continuum model(red plot). Figure 16 shows the 


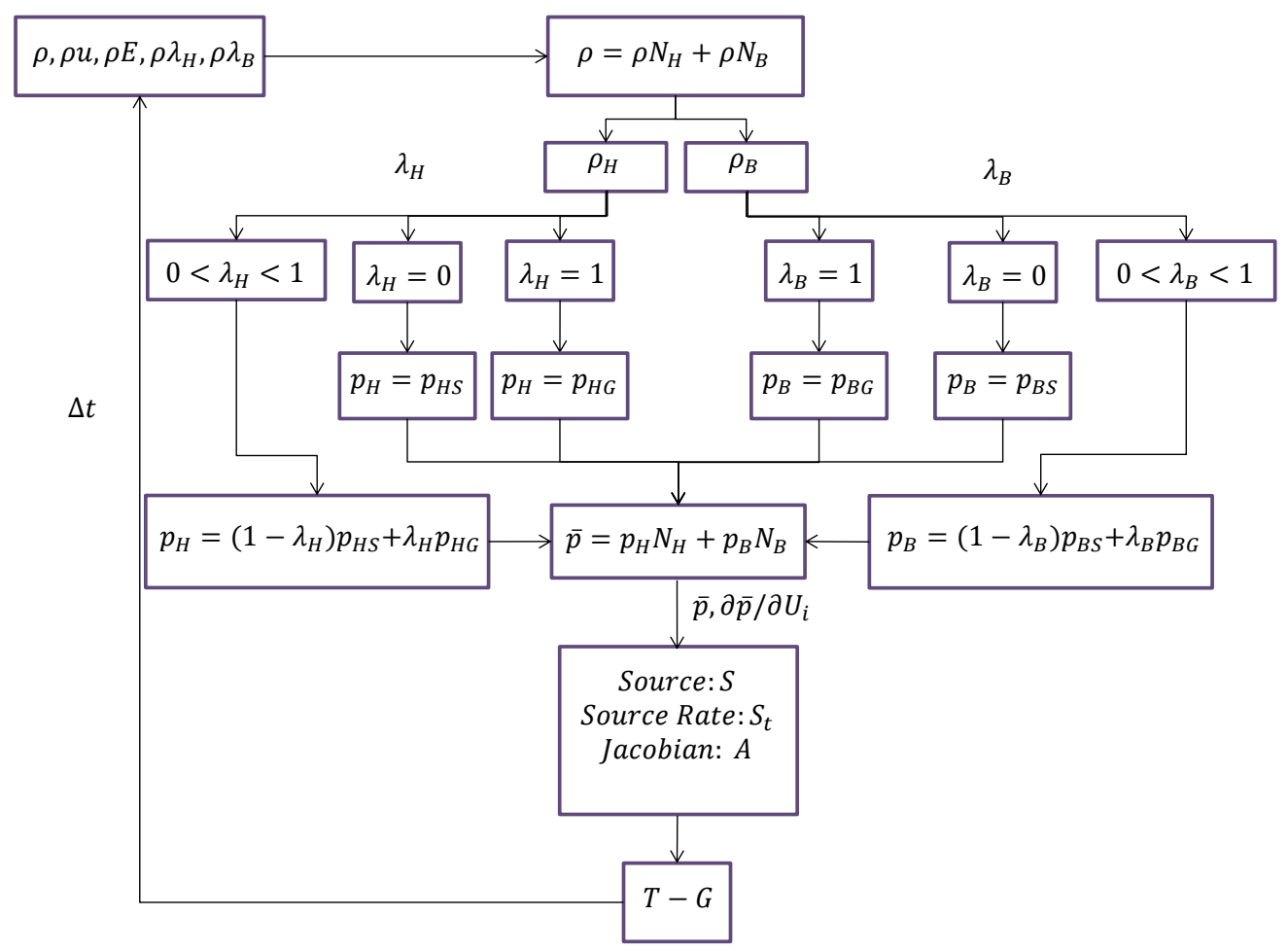

Figure 15. Multiscale modeling methodology.

Hugonoit and Figure 17 shows the Rayleigh line. By taking the microstructure of PBX 9501 into account, the Hugonoit has shifted corresponding to a reduction in Von Neumman pressure from $p_{V N}=0.59 \mathrm{Mbar}$ to $p_{V N}=0.57$ Mbar. The Rayleigh lines shown on Figure 17 differ by $3 \%$ leading to a differing shock wave speed. Both models are initiated with their corresponding Von Neumman points with numerical results for pressure shown on Figure 18 and Figure 19. The growth parameter and pressure exponent and equal for these results; i.e. $G_{H}=G_{B}$ and $b_{H}=b_{G}$. Both models are under the calculated Von Neumman pressure due to damping scheme described above. The pressures are directly compared at $t=1 \mu s$ on Figure 20 . It is clear from the plot that the multiscale model detonation wave travels at a faster speed than the continuum model wave speed.

\section{Conclusion}

Direct numerical simulation of shock propagation through PBXs was performed. Numerical result were obtained using a one-step second-order Taylor-Galerkin scheme. The numerical scheme was tested and agreed with exact solutions for SOD shock tube problem and the ZND detonation model. A Pressurebased reaction scheme based on the Ignition and Growth model was used with the Murnaghan and JWL equations of state for unreacted solid and fully reacted product respectively. The total pressure was found using an analytic mixture rule. Detonation of PBX 9404 was performed and all solutions showed a selfsustaining wave propagate through the domain. The peak pressure compared well with the Von Neumman pressure calculated from the Hugonoit and was under predicted due to damping. Initial work into multiscale modeling was presented and showed that modeling the microstructure leads to differences in Hugoniot data which corresponds to differing detonation wave speeds. Future work will include full order multiscale model with the microstructure explicitly modeled. 


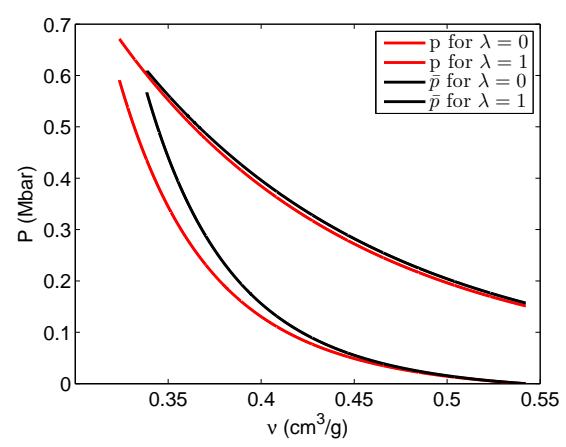

Figure 16. Hugonoit for continuum and multiscale model.

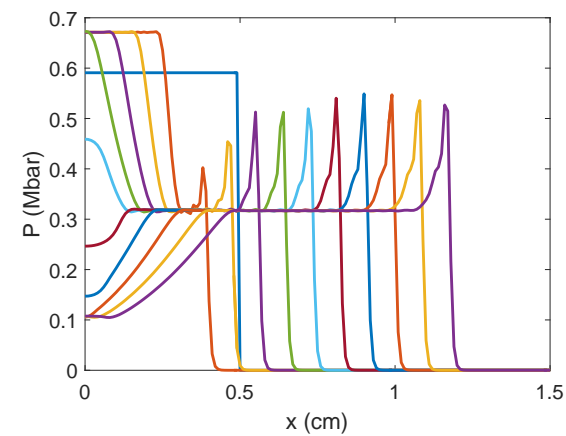

Figure 18. Numerical pressure results for continuum model.

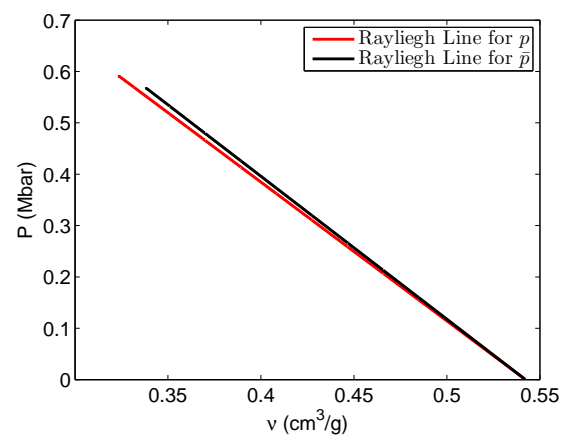

Figure 17. Rayleigh line for continuum and multiscale model.

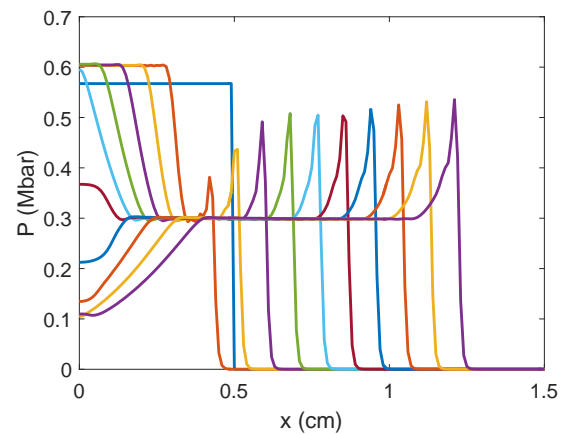

Figure 19. Numerical pressure results for multiscale model.

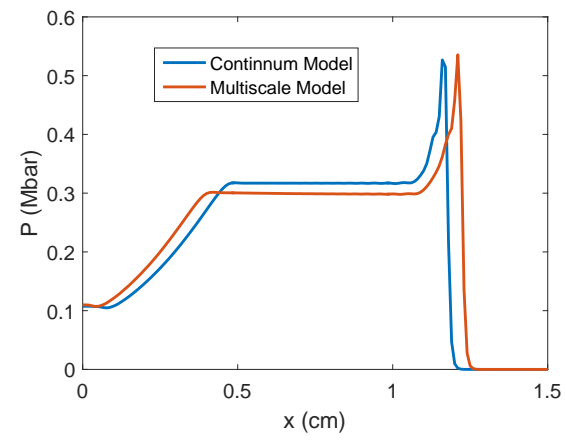

Figure 20. pressure results for continuum and multiscale model.

\section{Acknowledgments}

This material is based upon work supported by the National Science Foundation Graduate Research Fellowship Program Under DGE 1256260 and The Defense Threat Reduction Agency HDTRA1-13-1-0009.

\section{References}

${ }^{1}$ Fickett W, Davis W C (1979) Detonation. Berkeley

${ }^{2}$ Gogulya M F, Makhov M N, Dolgoborodov A Y, Brazhnikov M A, Arkhipov V I, Shchetinin V G (2004) Mechanical sensitivity and detonation parameters of aluminized explosives. Combustion, Explosion and Shock Waves 40(4):445-457.

${ }^{3}$ Duan Z P, Wen L J, Liu Y, Ou Z C, Huang F L, Zhang Z Y (2010) A Pore Collapse Model for Hot-spot Ignition in Shocked Multi-component Explosives. International Journal of Nonlinear Sciences and Numerical Simulation, 11(Supplement):19-24.

${ }^{4}$ Menikoff R (2006) Detonation waves in PBX 9501. Combustion Theory and Modelling 10(6):1003-1021. 
${ }^{5}$ Baer M R (2000) Computational modeling of heterogeneous reactive materials at the mesoscale. In Shock compression of condensed matter 505(1):27-34 AIP Publishing

${ }^{6}$ Baer M R, Trott W M (2002) Theoretical and experimental mesoscale studies of impact-loaded granular explosive and simulant materials. In Twelfth International Detonation Symposium.

${ }^{7}$ Benson D J, Conley P (1999) Eulerian finite-element simulations of experimentally acquired HMX microstructures. Modelling and Simulation in Materials Science and Engineering 7(3):333

${ }^{8}$ Brundage A L, Wixom R R, Tappan A S, Long G T (2009) Mesoscale simulations of shock initiation in energetic materials characterized by three-dimensional nanotomography. In Proceedings of the American Physical Society Topical Group on Shock Compression of Condensed Matter 1195(1):315-318 AIP Publishing

${ }^{9}$ Reaugh J E (2006) Multi-scale computer simulations to study the reaction zone of solid explosives. United States Department of Energy.

${ }^{10}$ Raymond W H, Garder A (1976) Selective damping in a Galerkin method for solving wave problems with variable grids. Monthly weather review, 104(12):1583-1590

${ }^{11}$ Brooks A N, Hughes T J (1982) Streamline upwind/Petrov-Galerkin formulations for convection dominated flows with particular emphasis on the incompressible Navier-Stokes equations. Computer methods in applied mechanics and engineering, 32(1):199-259

${ }^{12}$ Donea J (1984) A Taylor Galerkin method for convective transport problems. International Journal for Numerical Methods in Engineering, 20(1):101-119

${ }^{13}$ Baker A J, Kim J W (1987). A taylor weak statement algorithm for hyperbolic conservation laws. International journal for numerical methods in fluids, 7(5):489-520

${ }^{14}$ Löhner R, Morgan K, Zienkiewicz O C (1984) The solution of nonlinear hyperbolic equation systems by the finite element method. International Journal for Numerical Methods in Fluids, 4(11):1043-1063

${ }^{15}$ Nassehi V, Bikangaga J H (1993) A mathematical model for the hydrodynamics and pollutants transport in long and narrow tidal rivers. Applied mathematical modelling 17(8):415-422

${ }^{16}$ Quecedo M, Pastor M (2002) A reappraisal of Taylor Galerkin algorithm for drying wetting areas in shallow water computations. International journal for numerical methods in fluids 38(6):515-531.

${ }^{17}$ Lohner R, Morgan K, Peraire J, Vahdati M (1987) Finite element flux corrected transport (FEM-FCT) for the euler and Navier Stokes equations. International Journal for Numerical Methods in Fluids, 7(10):1093-1109

${ }^{18}$ McGuire R R, Tarver C M (1981) Chemical decomposition model for the thermal explosion of confined HMX, RDX and TNT explosives. In Seventh Symposium (International)on Detonation NSWC MP 82(334):56-64.

${ }^{19}$ Tarver C M, Tran T D (2004) Thermal decomposition models for HMX-based plastic bonded explosives. Combustion and Flame, 137(1):50-62.

${ }^{20}$ Henson B F, Smilowitz L, Romero J J, Asay B W (2009) Modeling thermal ignition and the initial conditions for internal burning in PBX 9501. In SHOCK COMPRESSION OF CONDENSED MATTER 2009: Proceedings of the American Physical Society Topical Group on Shock Compression of Condensed Matter, 1195(1):257-262

${ }^{21}$ Lee E L, Tarver C M (1980) Phenomenological model of shock initiation in heterogeneous explosives. Physics of Fluids (1958-1988) 23(12):2362-2372

${ }^{22}$ Souers P C, Anderson S, Mercer J, McGuire E, Vitello P (2000) JWL++: a simple reactive flow code package for detonation. Propellants, Explosives, Pyrotechnics 25(2):54-58

${ }^{23}$ Conley P A, Benson D J, Howe P M (1998) Microstructural effects in shock initiation. In Eleventh International Detonation Symposium ONR33300(5):768-780

${ }^{24}$ Menikoff R, Kober E (2000) Compaction waves in granular HMX. In Shock Compression of Condensed Matter-1999, 505(1):397-400

${ }^{25}$ Kury J W, Hornig H C, Lee E L, McDonnel J L, Ornellas D L, Finger M, Wilkins M L (1965) Metal acceleration by chemical explosives. In Fourth (International) Symposium on Detonation, ACR-126.

${ }^{26}$ Donea J, Huerta A (2003) Finite element methods for flow problems. John Wiley and Sons

${ }^{27}$ Dobratz B M, Crawford P C (1985) LLNL explosives handbook: properties of chemical explosives and explosive simulants. University of California, URCL-52997

${ }^{28}$ Handley C A (2011) Numerical Modelling of Two HMX-Based Plastic-Bonded Explosives at the Mesoscale. PhD Thesis, University of St. Andrews UK. 